\title{
Doppler-induced dynamics of fields in Fabry-Perot cavities with suspended mirrors
}

\author{
Malik Rakhmanov
}

\begin{abstract}
The Doppler effect in Fabry-Perot cavities with suspended mirrors is analyzed. The Doppler shift, which is intrinsically small, accumulates in the cavity and becomes comparable with or greater than the linewidth of the cavity if the cavity's finesse is high or its length is large. As a result, damped oscillations of the cavity field occur when one of the mirrors passes a resonance position. A formula for this transient is derived. It is shown that the frequency of the oscillations is equal to the accumulated Doppler shift and that the relaxation time of the oscillations is equal to the storage time of the cavity. Comparison of the predicted and the measured Doppler shifts is discussed, and application of the analytical solution for measurement of the mirror velocity is described. (C) 2001 Optical Society of America

OCIS codes: $120.2230,120.5700,120.7250,140.4780,280.3340$.
\end{abstract}

\section{Introduction}

Fabry-Perot cavities with lengths of several kilometers are utilized in laser gravitational wave detectors such as the Laser Interferometer Gravitational-Wave Observatory (LIGO). ${ }^{1}$ The mirrors in these FabryPerot cavities are suspended from wires and therefore are free to move along the direction of beam propagation. Ambient seismic motion excites the mirrors, causing them to swing like pendulums with frequencies of $\sim 1 \mathrm{~Hz}$ and amplitudes of $\sim 1 \mu \mathrm{m}$. To maintain the cavity on resonance, the Pound-Drever locking technique ${ }^{2}$ is used. During lock acquisition the mirrors frequently pass through resonances of the cavity. As one of the mirrors approaches a resonant position, the light in the cavity builds up. Immediately after the mirror passes a resonance position, a field transient in the form of damped oscillations occurs. This transient depends mostly on the cavity's length and finesse and on the relative velocity of the mirrors. Thus, careful examination of the transient reveals useful information about the cavity properties and the mirror motion.

When this research was performed, the author was with the LIGO Project, California Institute of Technology, 1200 East California Boulevard, Pasadena, California 91125. He is now with the Department of Physics, University of Florida, P.O. Box 118440, Gainesville, Florida 32611-0000. His e-mail address is malik@phys.ufl.edu.

Received 1 March 2000; revised manuscript received 16 January 2001.

0003-6935/01/121942-08\$15.00/0

(C) 2001 Optical Society of America
The oscillatory transient was observed in the past in several experiments with high-finesse FabryPerot cavities. The oscillations were recorded in the intensity of reflected light by Robertson et al. ${ }^{3}$ In that experiment the oscillations were used for measurements of the storage time of a Fabry-Perot cavity and its finesse. The oscillations were also observed in the intensity of transmitted light by An et al. ${ }^{4}$ In that experiment the oscillations were used for measurements of the cavity finesse and the mirror velocity. The transient was also studied by Camp et al. ${ }^{5}$ for applications to cavity-lock acquisition. This time the oscillations were observed in the Pound-Drever locking signal. Recently the transient was revisited by Lawrence et al. ${ }^{6}$ In that study both the cavitylength scans and the frequency scans were analyzed by use of all three signals: the intensities of the reflected and transmitted fields as well as the PoundDrever signal.

Although the transient has frequently been observed in experiments, its theory is far from being complete. It is known that the oscillations in the transient appear through the beatings of different field components in the cavity. However, different authors propose slightly different beat mechanisms. ${ }^{4,6}$ Moreover, it is not understood why the rate of the oscillations always increases in time and what causes this chirplike behavior.

In this paper it is shown that the transient can be explained by the Doppler effect that appears in a Fabry-Perot cavity with moving mirrors. The Doppler shift, which is intrinsically small, is amplified by the cavity and results in the modulation of the 


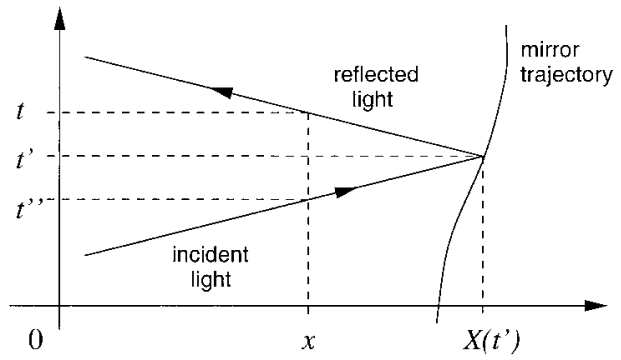

Fig. 1. Reflection of light off a moving mirror.

intracavity field. Based on this observation, we derive a simple formula for the transient and explain its chirplike behavior. In this approach the frequency of the oscillations can easily be found from the cavity parameters and the mirror velocity. The predictions based on the formula and on numerical simulations are compared with the measurements taken with the 40-m Fabry-Perot cavity of the Caltech prototype interferometer. In both cases good agreement is found.

Currently the transient is studied in connection with locking of the kilometer-sized Fabry-Perot cavities of LIGO interferometers. ${ }^{7}$ The analysis presented in this paper serves as a basis for calculations of the cavity parameters in these studies.

\section{Reflection of Light Off a Moving Mirror}

To set grounds for the analysis in this paper, we consider a simple process of reflection of light (electromagnetic wave) off a moving mirror. Let the mirror be moving along the $x$ axis with an arbitrary trajectory $X(t)$. Assume that the light is propagating along the positive $x$ direction and is normally incident upon the mirror. The wave front of the reflected wave observed at location $x$ and time $t$ is reflected by the mirror at some earlier time $t^{\prime}$, which, according to Fig. 1, satisfies the equation

$$
c\left(t-t^{\prime}\right)=X\left(t^{\prime}\right)-x .
$$

This equation defines time $t^{\prime}$ as an implicit function of $x$ and $t$.

Let the electric field of the incident and reflected waves be $\mathscr{E}_{\text {in }}(x, t)$ and $\mathscr{E}_{\text {ref }}(x, t)$, respectively. Because of continuity of the waves at the mirror surface, the two fields are related according to

$$
\mathscr{E}_{\text {ref }}(x, t)=\mathscr{E}_{\text {in }}\left[X\left(t^{\prime}\right), t^{\prime}\right]
$$

For simplicity we assume that the mirror is perfect (100\% reflective) and that no phase change occurs in the reflection.

Equations (1) and (2) allow us to calculate the wave reflected by a mirror moving along an arbitrary trajectory. Let the incident wave be plane and monochromatic:

$$
\mathscr{E}_{\text {in }}(x, t)=\exp [i(\omega t-k x)]
$$

where $\omega$ is the frequency and $k$ is the wave number $(k=\omega / c)$. Then the reflected wave is given by

$$
\mathscr{E}_{\text {ref }}(x, t)=\exp \left\{i\left[\omega t^{\prime}-k X\left(t^{\prime}\right)\right]\right\} .
$$

Substituting for $t^{\prime}$ from Eq. (1), we obtain that the electric field of the reflected wave is given by

$$
\mathscr{E}_{\text {ref }}(x, t)=\exp [i(\omega t+k x)] \exp \left[-2 i k X\left(t^{\prime}\right)\right] .
$$

The extra phase, $-2 k X\left(t^{\prime}\right)$, appears as a result of the continuity of the waves at the mirror surface and leads to two closely related effects. On the one hand, it gives rise to the phase shift of the reflected wave, which appears because the mirror position is changing. On the other hand, it gives rise to the frequency shift of the reflected wave, which appears because the mirror is moving. Indeed, the frequency of the reflected wave can be found as

$$
\omega^{\prime}(t)=\omega-2 k \frac{\mathrm{d} X}{\mathrm{~d} t^{\prime}} \frac{\partial t^{\prime}}{\partial t} .
$$

Note that $\mathrm{d} X / \mathrm{d} t$ is the instantaneous mirror velocity $v(t)$ and that

$$
\frac{\partial t^{\prime}}{\partial t}=\frac{c}{c+v\left(t^{\prime}\right)},
$$

which can be derived from Eq. (1). Combining Eqs. (6) and (7), we obtain the formula for the frequency of the reflected wave:

$$
\omega^{\prime}(t)=\frac{c-v\left(t^{\prime}\right)}{c+v\left(t^{\prime}\right)} \omega .
$$

At any given location the electric field oscillates at a very high frequency [ $\mathscr{E} \propto \exp (i \omega t)]$. It is convenient to remove the high-frequency oscillating factor $\exp (i \omega t)$ and consider only the slowly varying part of the wave:

$$
E(t) \equiv \mathscr{E}(x, t) \exp (-i \omega t) .
$$

The two amplitudes, $E_{1}(t)$ and $E_{2}(t)$, which correspond to the same wave but are defined at different locations $x$ and $x^{\prime}$, are related:

$$
E_{2}(t)=E_{1}(t-L / c) \exp (-i k L),
$$

where $L$ is the distance between the two locations $\left(L=x^{\prime}-x\right)$.

We now obtain a formula for the reflection off the moving mirror in terms of the slowly varying field amplitudes. One can do this by tracing the incident beam from the mirror surface back to the point with the coordinate $x$ :

$$
\mathscr{E}_{\text {ref }}(x, t)=\mathscr{E}_{\text {in }}\left(x, t^{\prime \prime}\right),
$$

where time $t^{\prime \prime}$ is further in the past and according to Fig. 1 is given by

$$
t^{\prime \prime}=2 t^{\prime}-t
$$


Equations (11) and (12) lead to the following relation between the field amplitudes:

$$
E_{\text {ref }}(t)=E_{\text {in }}\left(t^{\prime \prime}\right) \exp \left\{-2 i k\left[X\left(t^{\prime}\right)-x\right]\right\} .
$$

This formula is used below for calculations of fields in Fabry-Perot cavities with moving mirrors. For nonrelativistic mirror motion $(|v| \ll c)$ the frequency of the reflected light can be approximated as

$$
\omega^{\prime}(t) \approx\left[1-2 \frac{v\left(t^{\prime}\right)}{c}\right] \omega,
$$

which differs from the exact formula, Eq. (8), only in the second order in $v / c$.

\section{Doppler Shift in Fabry-Perot Cavities}

\section{A. Critical Velocity}

Fabry-Perot cavities of laser gravitational-wave detectors are long and have mirrors that can move. The Doppler shift in such cavities can be described as follows: Let the cavity length be $L$ and the transit time of light be $T$ :

$$
T=L / c .
$$

Assume that one of the mirrors is moving with constant velocity $v$. Then the frequency of light reflected off the moving mirror is Doppler shifted, and the shift in one reflection is

$$
\delta \omega \equiv \omega^{\prime}-\omega=-2 k v .
$$

Subsequent reflections make this frequency shift add, forming the progression

$$
\delta \omega, 2 \delta \omega, 3 \delta \omega, \ldots .
$$

Therefore the Doppler shift of light in the cavity accumulates with time.

A suspended mirror in such cavities moves little. Its largest velocity is typically of the order of a few micrometers per second. The corresponding Doppler shift is of the order of a few hertz, which is very small compared with the laser frequency $2.82 \times 10^{14}$ $\mathrm{Hz}$ for an infrared laser with wavelength $\lambda=1.06$ $\mu \mathrm{m}$. However, the linewidth of the long FabryPerot cavities of the laser gravitational wave detectors is also very small, typically of the order of $100 \mathrm{~Hz}$. Therefore the small Doppler shift, as it accumulates with time, can easily exceed the linewidth.

The characteristic time for light to remain in the cavity is the storage time, which is defined as $1 / e$ amplitude folding time:

$$
\tau=\frac{2 T}{\left|\ln \left(r_{a} r_{b}\right)\right|},
$$

where $r_{a}$ and $r_{b}$ are the amplitude reflectivities of the cavity mirrors. Then the Doppler shift accumulated within the storage time is

$$
|\delta \omega| \frac{\tau}{2 T}=\omega \frac{v \tau}{c T} .
$$

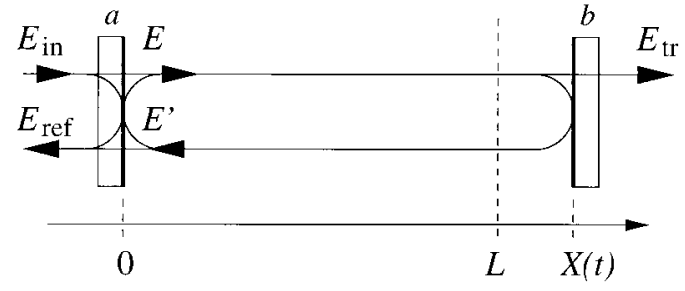

Fig. 2. Schematic diagram of a Fabry-Perot cavity with a moving mirror.

It becomes comparable with the linewidth of the cavity if the relative velocity of the mirrors is comparable with the critical velocity, defined as

$$
v_{\mathrm{cr}}=\frac{\lambda}{2 \tau \mathscr{F}^{2}} \approx \frac{\pi c \lambda}{4 L \mathscr{F}^{2}}
$$

where $\mathscr{F}$ is the finesse of the cavity:

$$
\mathscr{F}=\frac{\pi \sqrt{r_{a} r_{b}}}{1-r_{a} r_{b}} \text {. }
$$

Note that the mirror moving with the critical velocity passes the width of a resonance within the storage time. These qualitative arguments show that the Doppler effect becomes significant if the time for a mirror to move across the width of a resonance is comparable with or less than the storage time of the cavity.

\section{B. Equation for Fields in a Fabry-Perot Cavity}

The response of Fabry-Perot cavities is usually expressed in terms of amplitudes of the electromagnetic field circulating in the cavity. The equation for the dynamics of this field can be derived as follows: Assume, for simplicity, that one of the mirrors (input mirror) is at rest and the other (end mirror) is freely swinging. Let the trajectory of this mirror be $X(t)$. It is convenient to separate the constant and the variable parts of the mirror trajectory:

$$
X(t)=L+x(t) .
$$

In Fabry-Perot cavities of gravitational-wave detectors, $L$ is of the order of a few kilometers and $x$ is of the order of a few micrometers. Without loss of generality we can assume that cavity length $L$ is equal to an integer number of wavelengths and therefore $\exp (-2 i k L)=1$.

Let the amplitude of the input laser field be $E_{\text {in }}(t)$ and the amplitudes of the fields inside the cavity be $E(t)$ and $E^{\prime}(t)$, both defined at the reflective surface of the input mirror, as shown in Fig. 2. Then the equation for reflection off the end mirror can be written as follows:

$$
E^{\prime}(t)=-r_{b} E(t-2 T) \exp [-2 i k x(t-T)],
$$


where $r_{b}$ is the amplitude reflectivity of the end mirror. A similar equation can be written for the reflection off the front mirror:

$$
E(t)=-r_{a} E^{\prime}(t)+t_{a} E_{\text {in }}(t),
$$

where $t_{a}$ is the transmissivity of the front mirror.

Finally, the amplitudes of the transmitted and the reflected fields are given by

$$
\begin{aligned}
E_{\mathrm{tr}}(t) & =t_{b} E(t-T), \\
E_{\mathrm{ref}}(t) & =r_{a} E_{\mathrm{in}}(t)+t_{a} E^{\prime}(t),
\end{aligned}
$$

where $t_{b}$ is the transmissivity of the end mirror. Note that the reflected field is a superposition of the intracavity field leaking through the front mirror and the input laser field reflected by the front mirror, as shown in Fig. 2.

It is convenient to reduce Eqs. (23) and (24) to one equation with one field:

$$
E(t)=t_{a} E_{\text {in }}(t)+r_{a} r_{b} E(t-2 T) \exp [-2 i k x(t-T)] .
$$

Further analysis of field dynamics in the FabryPerot cavities is based on this equation.

\section{Transient Caused by Mirror Motion}

The mirrors in Fabry-Perot cavities of laser gravitational-wave detectors are suspended from wires and can swing like pendulums with frequencies of $\sim 1 \mathrm{~Hz}$. The amplitude of such motion is of the order of a few micrometers. During the swinging motion, the mirrors frequently pass through resonances of the cavity. Each passage through a resonance gives rise to the field transient in the form of damped oscillations. Such a transient can be described in terms of the complex amplitude of the cavity field as follows: For the entire time when the mirror moves through the width of a resonance (a few milliseconds), its velocity can be considered constant, and its trajectory can be approximated as linear: $x=v t$. Often the amplitude of the incident field is constant: $E_{\text {in }}(t)=A$. Then the amplitude of the intracavity field satisfies the equation

$$
E(t)=t_{a} A+r_{a} r_{b} E(t-2 T) \exp [-2 i k v(t-T)],
$$

which is a special case of Eq. (27).

Numerical solutions of Eq. (27) can easily be obtained with a computer. Examples of the numerical solution with the parameters of LIGO 4-km FabryPerot cavities are shown in Fig. 3. Such numerical solutions provide an accurate description for the field transient but give little insight into the physics of the process. Therefore it is worthwhile to obtain an approximate analytical solution for this equation.


Fig. 3. Modeled response of the LIGO 4-km Fabry-Perot cavity (finesse 205). The two curves correspond to the slow (top) and the fast (bottom) motions of the mirror $\left(v_{\mathrm{cr}}=1.48 \times 10^{-6} \mathrm{~m} / \mathrm{s}\right)$.

\section{A. Approximate Solution for the Transient}

An approximate solution can be derived as follows: A general solution of Eq. (28) can be represented as a sum:

$$
E(t)=C(t)+D(t) .
$$

Here $C(t)$ is a particular solution of the nonhomogeneous equation and $D(t)$ is a general solution of the homogeneous equation:

$$
\begin{aligned}
& C(t)-r_{a} r_{b} C(t-2 T) \exp [-2 i k v(t-T)]=t_{a} A, \\
& D(t)-r_{a} r_{b} D(t-2 T) \exp [-2 i k v(t-T)]=0 .
\end{aligned}
$$

Both amplitudes, $C(t)$ and $D(t)$, change little during one round trip. For the $C$ field the approximation $C(t-2 T) \approx C(t)$ yields the solution

$$
C(t) \approx \frac{t_{a} A}{1-r_{a} r_{b} \exp (-2 i k v t)},
$$

which is generally known as the adiabatic field. [Here we have also made the approximation that $v(t-T) \approx v t$. $\quad$ The adiabatic component was introduced empirically by Yamamoto. ${ }^{7}$

For the $D$ field the approximation $D(t-2 T) \approx D(t)$ yields only a trivial solution: $D(t)=0$. Fortunately, the equation for the $D$ field can be solved exactly. A trial solution for $t>0$ is

$$
D(t)=D_{0}\left(r_{a} r_{b}\right)^{t / 2 T} \exp [i \phi(t)],
$$

where $D_{0}$ is the value of the $D$ field at time $t=0$ and $\phi(t)$ is an arbitrary phase. Then Eq. (31) reduces to the equation for the phase

$$
\phi(t)=\phi(t-2 T)-2 k v(t-T) .
$$


Its solution, up to an additive constant, is

$$
\phi(t)=-\frac{k v}{2 T} t^{2}
$$

Thus we obtain the solution for the $D$ field:

$$
D(t)=D_{0} \exp \left(-\frac{t}{\tau}-i \frac{k v}{2 T} t^{2}\right)
$$

where $\tau$ is the cavity storage time defined in Eq. (18). This expression is valid for $t>0$ and describes the phase modulation of the cavity field that is due to the Doppler effect. The constant $D_{0}$ can be found from the asymptotic behavior of the field ${ }^{8}$ and is given by

$$
D_{0}=t_{a} A\left(\frac{i \pi}{2 k v T}\right)^{1 / 2} \exp \left(\frac{i T}{2 k v \tau^{2}}\right) \text {. }
$$

Equation (36) shows that the $D$ field oscillates with the frequency that increases linearly with time:

$$
\Omega(t) \equiv\left|\frac{\mathrm{d} \phi}{\mathrm{d} t}\right|=\frac{k|v|}{T} t .
$$

Note that the frequency of the oscillations is equal to the accumulated Doppler shift:

$$
\Omega(t)=|\delta \omega| \frac{t}{2 T},
$$

where $\delta \omega$ is the frequency shift that occurs in one reflection off the moving mirror [Eq. (16)].

Combining Eqs. (32) and (36) we obtain the formula for the transient:

$$
\begin{aligned}
E(t)= & \frac{t_{a} A}{1-r_{a} r_{b} \exp (-2 i k v t)} \\
& +D_{0} \exp \left(-\frac{t}{\tau}-i \frac{k v}{2 T} t^{2}\right) .
\end{aligned}
$$

Thus the transient, which occurs during a passage of the mirror through a resonance, is caused by the Doppler effect amplified by the cavity. The frequency of oscillations increases linearly in time with the rate proportional to the mirror velocity (Fig. 4).

Comparison of the approximate analytical solution given by Eq. (40) with the numerical simulations based on Eq. (28) shows that the two solutions agree well in the region past the resonance $(t \gg T)$. However, the two solutions differ substantially in the region near the center of the resonance $(t \approx 0)$. This is so because the center of the resonance is the boundary of the validity of the approximate analytical solution.

The above analysis leads to the following explanation of the oscillatory transient: As the mirror approaches the resonance position $(x=0)$, the light builds rapidly in the cavity. At the time when the mirror passes the center of the resonance, a substantial amount of light accumulates in the cavity. From this moment on, the light stored in the cavity ( $D$ component) decays according to the exponential law,

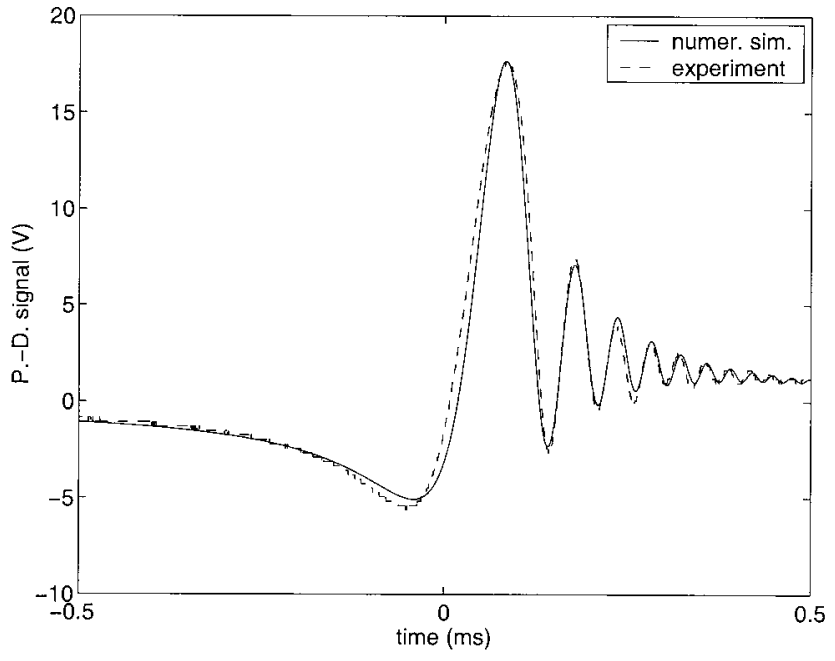

Fig. 4. Transient response of the Fabry-Perot cavity of the Caltech $40-\mathrm{m}$ prototype interferometer $\left(v / v_{\text {cr }}=1.93\right)$.

and its frequency shifts continuously as a result of the Doppler effect. At the same time there is a constant influx of the new light from the laser $(C$ component). The new light is not affected by the Doppler shift and therefore evolves according to the usual adiabatic law.

\section{B. Observation of the Transient through Beats}

The small frequency shifts of the light circulating in the cavity are usually observed through beats. There are several beat mechanisms that take place in Fabry-Perot cavities with moving mirrors. Here we describe the three most frequently encountered beat mechanisms in detail.

The Doppler-induced oscillations of the intracavity field can be observed in the intensity of the transmitted field. The above analysis shows that the Doppler effect gives rise to phase modulation of the $D$ field. As a result, cavity field $E$, which is the sum of the $D$ and $C$ fields, becomes amplitude modulated. This amplitude modulation can be observed as the intensity modulation of the field transmitted through the cavity. According to Eqs. (25) and (29) the intensity of the transmitted field is proportional to

$$
|E(t)|^{2}=|C(t)|^{2}+|D(t)|^{2}+2 \operatorname{Re}[C(t) * D(t)],
$$

where an asterisk stands for complex conjugation. Note that neither $|C(t)|^{2}$ nor $|D(t)|^{2}$ is an oscillating function. Therefore the oscillations come from the last term, which represents a beating between $D$ and $C$ components of the intracavity field.

Similarly, the oscillations of the intracavity field can be observed in the intensity of the reflected field. According to Eqs. (23) and (26), the amplitude of the reflected field can be found as

$$
E_{\mathrm{ref}}(t)=\left[\left(r_{a}^{2}+t_{a}^{2}\right) E_{\mathrm{in}}(t)-t_{a} E(t)\right] / r_{a} \text {. }
$$


For high-finesse cavities $\left(r_{a} \approx 1\right)$ with low losses $\left(r_{a}{ }^{2}+t_{a}{ }^{2} \approx 1\right)$ the complex amplitude of the reflected field can be approximated as

$$
E_{\text {ref }}(t)=E_{\text {in }}(t)-t_{a} E(t) .
$$

Then the intensity of the reflected light is given by

$$
\left|E_{\text {ref }}(t)\right|^{2}=\left|E_{\text {in }}(t)\right|^{2}+t_{a}{ }^{2}|E(t)|^{2}-2 t_{a} \operatorname{Re}\left[E_{\text {in }}(t) * E(t)\right] .
$$

The second term on the right-hand side of Eq. (44) represents the amplitude modulation of the intracavity field as described in Eq. (41). The last term represents a beating of the intracavity field transmitted through the front mirror and the input laser field promptly reflected by the front mirror. Both terms give rise to the oscillations in the intensity of the reflected field. Therefore the decay of the reflected intensity is described by the double exponential function with two decay times, $\tau$ and $\tau / 2$, as was noted by Robertson et al. ${ }^{3}$

The oscillations can also be observed in the PoundDrever signal, which requires that optical sidebands be imposed on the light that is incident upon the cavity. In this case the signal is obtained from beating of the carrier reflected field with the sideband reflected fields. Inasmuch as the carrier field propagates in the cavity, it becomes Doppler shifted as a result of the motion of the cavity mirrors. The sideband fields are promptly reflected by the front mirror of the cavity. Therefore their amplitudes are proportional to the amplitude of the incident carrier field. Then the signal can be approximated by the formula

$$
V(t)=-\operatorname{Im}\left[\exp (i \gamma) E_{\text {in }}(t)^{*} E(t)\right],
$$

where $\gamma$ is the phase of a local oscillator in the optical heterodyne detection.

If the amplitude of the input laser field is constant $\left[E_{\text {in }}(t)=A\right]$, then the Pound-Drever signal becomes a linear function of the cavity field:

$$
\begin{aligned}
V(t) & =-A \operatorname{Im}[\exp (i \gamma) E(t)], \\
& =-A \operatorname{Im}\{\exp (i \gamma)[C(t)+D(t)]\} .
\end{aligned}
$$

Because the $C$ component is a monotonic function, the oscillations come from the $D$ component only. Unlike the signals derived from the intensity of the transmitted or reflected fields, the Pound-Drever signal is linearly proportional to the amplitude of the intracavity field and therefore presents a direct way to observe the oscillations.

\section{Experimental Analysis of the Transient}

The measurements of the oscillatory transient analyzed in this paper were taken with the 40-m FabryPerot cavity of the LIGO-prototype interferometer at Caltech. The experimental setup was previously described by Camp et al. ${ }^{5}$ Figure 4 shows the PoundDrever signal of the 40-m Fabry-Perot cavity recorded with a digital oscilloscope (dashed curve).
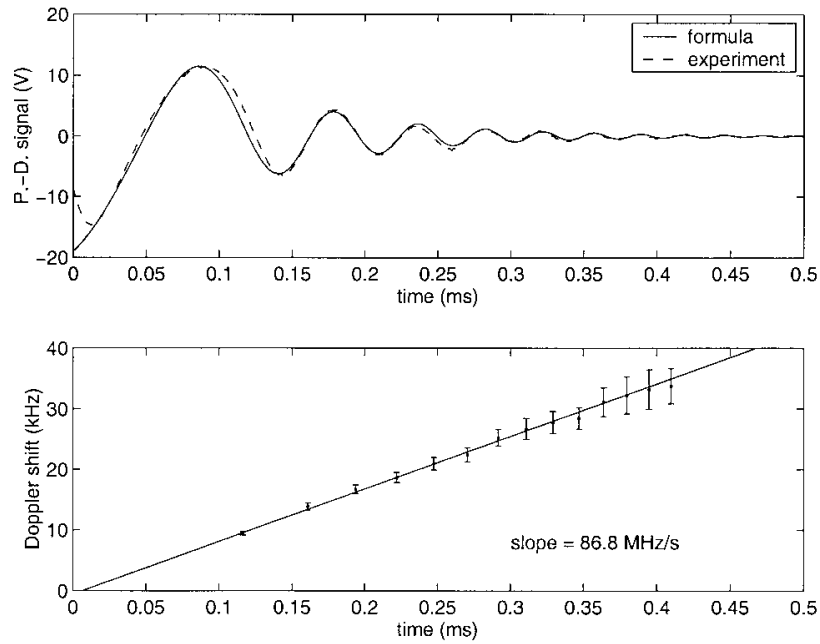

Fig. 5. Top, theoretical prediction (solid curve) and measurement (dashed curve) of the adjusted Pound-Drever (P.-D.) signal. Bottom, measured Doppler shift $\bar{v}_{n}$ and linear fit $\bar{v}(t)$.

The theoretical prediction shown in the same figure (solid curve) is obtained by numerical simulations of the intracavity field by use of Eq. (28). After adjustment of the demodulation phase $(\gamma \approx-0.28 \mathrm{rad})$, good agreement between the theoretical and the experimental curves was achieved. It is important to note that the mirror velocity $\left(v \approx 5.5 \times 10^{-6} \mathrm{~m} / \mathrm{s}\right)$ used for the numerical simulations was not a fit parameter. It was obtained from the interpolation of the mirror trajectory by the optical vernier technique. ${ }^{9}$

The formula for the transient, Eq. (40), can be used for extracting the cavity parameters from the PoundDrever signal. In such an analysis it is convenient to remove the adiabatic component from the PoundDrever signal. The result is a function similar to $D(t)$, which is given by

$$
\begin{aligned}
V_{D}(t)= & -A\left|D_{0}\right| \exp \left(-\frac{t-t_{0}}{\tau}\right) \\
& \times \sin \left[\gamma+\delta-\frac{k v}{2 T}\left(t-t_{0}\right)^{2}\right] .
\end{aligned}
$$

Here we have introduced $t_{0}$, the time when the mirror passes a center of the resonance, and $\delta=\arg D_{0}$. The measured Pound-Drever signal (with the adiabatic component removed) and the theoretical prediction based on this formula are shown in Fig. 5 (top).

\section{A. Measurement of the Cavity Finesse}

The oscillatory transient can be used for measurements of the cavity finesse. The present approach is based on the exponential decay of the Pound-Drever signal. One can find the finesse by studying the absolute value of the adjusted Pound-Drever signal:

$$
\left|V_{D}(t)\right| \propto \exp (-t / \tau)
$$

Indeed, by fitting the exponential function to the envelope of the oscillations $\left|V_{D}(t)\right|$, one can find the stor- 
age time of the cavity, $\tau$, and therefore the cavity's finesse:

$$
\mathscr{F}=\frac{\pi}{2 \sinh (T / \tau)} .
$$

Applied to the data shown in Fig. 4, this method yields the following value for the finesse of the Caltech 40-m Fabry-Perot cavity:

$$
\mathscr{F}=1066 \pm 58 .
$$

This result is close to the one previously obtained from the measurement of the mirror reflectivities (F्F $\approx 1050$ ). The present approach to measuring cavity storage time is similar to the one described by Robertson et al. ${ }^{3}$

\section{B. Measurement of the Mirror Velocity}

The oscillatory transient can also be used for measurements of the mirror velocity. The present approach is based on the linear shift of the frequency of the Pound-Drever signal. One can find the velocity by studying either the peaks or the zero crossings of the adjusted Pound-Drever signal, $V_{D}(t)$.

Let the times for the zero crossings be $t_{n}$, where $n$ is an integer. The values for $t_{n}$ are defined by the functional form of the adjusted Pound-Drever signal [Eq. (48)], and are given by

$$
\frac{k v}{2 T}\left(t_{n}-t_{0}\right)^{2}=\pi n+\gamma+\delta .
$$

This relation depends on the demodulation phase $\gamma$, which is not always known in the experiment. However, the difference

$$
\frac{k v}{2 T}\left[\left(t_{n+1}-t_{0}\right)^{2}-\left(t_{n}-t_{0}\right)^{2}\right]=\pi
$$

does not depend on the demodulation phase and therefore is more suitable for this analysis. Define the spacings between the zero crossings, $\Delta t_{n}$, and the positions of their midpoints, $\bar{t}_{n}$, as follows:

$$
\begin{aligned}
\Delta t_{n} & =t_{n+1}-t_{n}, \\
\bar{t}_{n} & =\left(t_{n}+t_{n+1}\right) / 2 .
\end{aligned}
$$

Then the average frequencies of the oscillations of $V_{D}(t)$ can be defined as

$$
\bar{v}_{n}=\frac{1}{2 \Delta t_{n}} .
$$

Using the identity given by Eq. (53), we can show that the average frequencies satisfy the equation

$$
\bar{v}_{n}=\frac{v}{\lambda T}\left(\bar{t}_{n}-t_{0}\right) .
$$

This equation is a discrete analog of the continuous evolution [Eq. (38)].

If the times $t_{n}$ correspond to the peaks and not to the zero crossings of the signal, the predicted average frequencies become

$$
\bar{v}_{n}=\frac{v}{\lambda T}\left(\bar{t}_{n}-t_{0}\right)+\delta \bar{v}_{n},
$$

where $\delta \bar{v}_{n}$ is a small correction that accounts for the exponential decay of the signal that is present in Eq. (48). The correction can be found from Eq. (48) by use of a perturbation expansion in powers of $T / \tau$. In the lowest order, it is given by

$$
\delta \bar{v}_{n}=-\frac{4 \lambda v T\left(\bar{t}_{n}-t_{0}\right)^{2}}{\pi^{2} \tau\left[16 v^{2}\left(\bar{t}_{n}-t_{0}\right)^{4}-\lambda^{2} T^{2}\right]} .
$$

Such a correction becomes significant only if the oscillations are close to being critically damped.

In general, the zero crossings can be affected by the subtraction of the adiabatic component. Therefore we prefer to use the peaks of the signal. The peak positions $t_{n}$ are found from the measured PoundDrever signal, which is shown in Fig. 5 (top). Because the oscillations are far from being critically damped, the correction $\delta \bar{v}_{n}$ can be neglected. In this experiment, the first-order correction is much less than the error in determination of the average frequencies. As a result the measured values of the average frequencies $v_{n}$ appear close to the linear function [Eq. (57)], as can be seen in Fig. 5 (bottom). Therefore we can apply a linear fit to the data:

$$
\bar{v}(t)=a t+b,
$$

where $a$ and $b$ are the slope and the intercept of the linear function. The least-squares adjustment of the fit gives the following values for these parameters:

$$
\begin{aligned}
& a=(86.8 \pm 0.6) \times 10^{6} \mathrm{~Hz} / \mathrm{s}, \\
& b=(-0.5 \pm 1.0) \times 10^{3} \mathrm{~Hz} .
\end{aligned}
$$

The slope is related to the mirror velocity, and the intercept is related to the time when the mirror passes through the center of the resonance:

$$
\begin{aligned}
v & =\lambda T a, \\
t_{0} & =-b / a .
\end{aligned}
$$

From these relations we obtain

$$
\begin{aligned}
v & =(5.7 \pm 0.4) \times 10^{-6} \mathrm{~m} / \mathrm{s}, \\
t_{0} & =(0.6 \pm 1.2) \times 10^{-5} \mathrm{~s} .
\end{aligned}
$$

The errors are due to uncertainty in the peak positions, which are limited in this measurement by the resolution of the oscilloscope.

\section{Conclusions}

The Doppler effect in Fabry-Perot cavities with suspended mirrors can be significant and manifests itself in the oscillations of the field transient, which can be directly observed in the Pound-Drever signal. The 
transient can be used for accurate measurements of the cavity finesse and the mirror velocities. The formula for the transient, implemented in real-time computer simulations, can be used in lock-acquisition algorithms.

The analysis presented in this paper explains the chirplike behavior of the transient and leads to a simple formula for the frequency of the chirp. However, the approximate analytical solution given in this paper describes only the ringdown part of the transient. The buildup part has yet to be explained. Also, it is not clear at the present time why oscillations appear always after the mirror passes the center of the resonance and not before.

I thank Guoan $\mathrm{Hu}$ for assistance with the experiment and Hiro Yamamoto and Matt Evans for helpful discussions. I also thank Barry Barish and other scientists of the LIGO project: Rick Savage, David Shoemaker, and Stan Whitcomb, for their suggestions about the manuscript during the draft process. Finally, I thank David Reitze and David Tanner of the University of Florida for discussions of the transient and their comments on the paper. This research was supported by the National Science Foundation under cooperative agreement PHY-9210038.

\section{References}

1. A. Abramovici, W. E. Althouse, R. W. Drever, Y. Gürsel, S. Kawamura, F. J. Raab, D. Shoemaker, L. Sievers, R. E.
Spero, K. S. Thorne, R. E. Vogt, R. Weiss, S. E. Whitcomb, and M. E. Zucker, "LIGO: the Laser Interferometer Gravitational-Wave Observatory," Science 256, 325-333 (1992).

2. R. W. P. Drever, J. L. Hall, F. V. Kowalski, J. Hough, G. M. Ford, A. J. Munley, and H. Ward, "Laser phase and frequency stabilization using an optical resonator," Appl. Phys. B 31, 97-105 (1983).

3. N. A. Robertson, K. A. Strain, and J. Hough, "Measurements of losses in high reflectance mirrors coated for $\lambda=514.5 \mathrm{~nm}$," Opt. Commun. 69, 345-348 (1989).

4. K. An, C. Yang, R. R. Dasari, and M. S. Feld, "Cavity ring-down technique and its application to the measurement of ultraslow velocities," Opt. Lett. 20, 1068-1070 (1995).

5. J. Camp, L. Sievers, R. Bork, and J. Heefner, "Guided lock acquisition in a suspended Fabry-Perot cavity," Opt. Lett. 20, 2463-2465 (1995).

6. M. J. Lawrence, B. Willke, M. E. Husman, E. K. Gustafson, and R. L. Byer, "Dynamic response of a Fabry-Perot interferometer,” J. Opt. Soc. Am. B 16, 523-532 (1999).

7. H. Yamamoto, "Fringe structure of LIGO Hanford $2 \mathrm{~km}$ FabryPerot cavity," LIGO Tech. Rep. G990130 (California Institute of Technology, Pasadena, Calif., 1999).

8. M. Rakhmanov, "Dynamics of laser interferometric gravitational wave detectors," Ph.D. dissertation (California Institute of Technology, Pasadena, Calif., 2000).

9. M. Rakhmanov, M. Evans, and H. Yamamoto, "An optical vernier technique for in situ measurement of the length of long Fabry-Perot cavities," Meas. Sci. Technol. 10, 190-194 (1999). 